\title{
REVIEW
}

\section{Proteomic profiling in schizophrenia: enabling stratification for more effective treatment}

\author{
Paul C Guest*1, Daniel Martins-de-Souza2,3,4, Emanuel Schwarz', Hassan Rahmoune', Murtada Alsaif', Jakub Tomasik', \\ Christoph W Turck² and Sabine Bahn,
}

\begin{abstract}
Schizophrenia is a heterogeneous psychiatric disorder characterized by an array of clinical manifestations. Although the best known manifestations include serious effects on mood and behavior, patients can also display co-morbidities, including immune system or metabolic abnormalities. Thorough characterization of these conditions using proteomic profiling methods has increased our knowledge of these molecular differences and has helped to unravel the complexity and heterogeneity of this debilitating condition. This could lead to patient stratification through characterization of biochemically different subtypes of the disease. In addition, proteomic methods have recently been used for molecular characterization of the mechanism of action of antipsychotic medications in both preclinical models and patients. This has resulted in identification of molecular panels that show some promise for prediction of response or for monitoring treatment outcome. This review describes how proteomic profiling methods can impact the future of schizophrenia diagnosis and therapeutics, and facilitate personalized medicine approaches for more effective treatment management of schizophrenia patients.
\end{abstract}

\section{Introduction \\ Fewer than $50 \%$ of schizophrenia patients respond to initial treatment with antipsychotic medications [1]. This is mainly due to the fact that there is not sufficient understanding of the underlying pathophysiology to aid diagnosis or treatment selection [2]. Furthermore, the traditional treatment approach usually involves random}

\footnotetext{
*Correspondence: pg110@cam.ac.uk

'Department of Chemical Engineering and Biotechnology, University of

Cambridge, Tennis Court Road, Cambridge, CB2 1QT, UK

Full list of author information is available at the end of the article
}

selection and switching of drugs multiple times to achieve an adequate response. This has resulted in high drug attrition and lack of efficacy of blockbuster drugs in patient subpopulations. Therefore, reliable tests with a biological rationale to guide treatment selection are needed. Despite almost a century of research, the diagnosis of schizophrenia still relies on the subjective assessment of symptoms by clinicians according to classifications listed in the Diagnostic and Statistical Manual of Mental Disorders, 4th Edition, or the International Statistical Classification of Diseases and Related Health Problems, 10th Revision. Also, current diagnoses are biased towards detection of symptoms of psychosis. Schizophrenia symptoms are generally classified as positive (psychosis, hallucinations, delusions), negative (anhedonia, dysphoria, affective flattening) or cognitive (low attention, memory, executive functions). However, it is not known whether specific symptoms are linked to defined pathophysiologies [3]. As a result, there has been a recent shift towards the study and identification of molecular biomarkers in psychiatric disorders, using techniques such as proteomics [4].

Proteomic techniques can be used as a non-biased screening approach and analyses of postmortem brain tissues from schizophrenia patients have provided insights into the pathways affected in the disease. It is anticipated that the use of biomarkers identified in these studies can lead to improved diagnosis through better classification of patients, to more effective treatment of patients by identification of those who are most likely to respond to specific medications, and to development of more efficacious drugs that target the relevant molecular pathways.

Recent studies have established a role for neuroinflammation in schizophrenia, as shown by increased serum concentrations of pro-inflammatory cytokines and chronic humoral responses [5]. Moreover, proteomic studies of postmortem brain tissue have found differences in immune-related proteins [6]. Schizophrenia has also been associated with peripheral manifestations related to metabolic or hormonal dysregulation, including dyslipidemia, hyperinsulinemia and type 2 diabetes mellitus $[7,8]$. 
Although these effects can result from administration of antipsychotic medications, they have also been observed prior to disease development when no antipsychotics were used [8]. Furthermore, proteomic analyses of postmortem brain tissues from schizophrenia patients have identified effects on proteins involved in regulation of neuronal and synaptic function [9-13] and alterations in glucose metabolism and insulin signaling pathways $[14,15]$. This suggests a link between the brain and periphery in the onset and development of the disease. Likewise, there have been reports of hypothalamic-pituitary-adrenal (HPA) axis disturbance in schizophrenia, which has been linked to abnormal insulin signaling $[16,17]$.

This review will address the study of schizophrenia based on differences in proteomic signatures in the central nervous system and peripheral tissues related to dysregulation in inflammatory, metabolic and hormonal pathways. Taken together, these findings suggest the presence of distinct molecular endophenotypes as risk factors for schizophrenia. We will also review studies that have attempted to identify proteomic profiles that can predict response to antipsychotic medications. We hope to demonstrate that proteomic research can contribute to de-convoluting the complexity of schizophrenia, from symptom-based concepts to a biological understanding for the ultimate application in personalized medicine approaches.

\section{The need for proteomics-based biomarkers in schizophrenia}

The development of biomarkers is constantly advancing with the aim of applying these in diagnostic studies and clinical trials. Regulatory health authorities such as the Food and Drug Administration (FDA) now consider biomarkers to be essential in the pharmaceutical industry for antipsychotic drug discovery [18]. In line with this, they have called for modernization of methods and technologies to facilitate the delivery of more efficacious and safer drugs [19]. According to the FDA guidance for pharmaceutical companies on pharmacogenomic data, biomarkers must be categorized into three classes: (1) exploratory biomarkers, (2) probable valid biomarkers, and (3) known valid biomarkers [20]. For the first class, there must be scientific evidence. The transition to the second class requires that a biomarker can be measured in a test system with well-established performance characteristics and that there is established evidence that explains the biological, pharmacological, toxicological or clinical significance of the results. A biomarker will achieve the third class if the results can be replicated in studies involving a large number of patients at different sites, laboratories or agencies in cross-validation experiments.

One strategy for biomarker qualification that is receiving increasing interest is through their co-development with drugs [21]. This requires that biomarker use is limited to applications involving the corresponding drug. This approach was first described by the FDA in a white paper guidance that stipulated that increased knowledge about the biology of a biomarker and a robust association between the biomarker signal and the clinical result will lead to a more efficient drug development process with a higher probability of success [21]. Thus, inclusion of biomarkers into the clinical development pipeline will only be achieved by a rigorous scientific approach, which includes standardized operating procedures for patient selection, sample collection, laboratory analysis and data processing. Also, early interaction with the appropriate regulatory agencies is essential to ensure that studies are designed and biomarker tests are carried out appropriately.

However, this is not a simple task. The identification of biomarkers for psychiatric disorders is challenging due to the overlap of symptoms across different disorders and the marked heterogeneity of these symptoms in affected individuals. In addition, there is currently a poor understanding of the underlying causes of the disease at the molecular level. However, emerging proteomic platforms have facilitated the identification of biomarker candidates by simultaneous measurement of hundreds or thousands of molecules in non-hypothesis driven profiling studies. It is expected that biomarkers that are associated with the disease state or with the mechanism of action of psychiatric medications will lead to improved diagnosis and pave the way for more effective treatment of patients.

\section{Proteomic technologies used in schizophrenia research}

Early proteomic studies in psychiatry have used twodimensional gel electrophoresis followed by mass spectrometry (MS) for comparative global analyses. Developments in MS instrumentation and techniques have allowed the establishment of high-throughput proteomic techniques. The basic idea of shotgun proteomics is to digest the proteome of interest using specific enzymes and identify the resulting peptides by sensitive and accurate MS analyses. Depending on the complexity of a given proteome, pre-fractionation steps may be required using techniques such as subcellular fractionation, liquid chromatography and gel-based techniques. A range of techniques can be used for quantification of shotgun MS results, such as in vivo or in vitro stable isotope labeling, isobaric tag for relative and absolute quantification, and label-free approaches [22]. Shotgun MS-based studies [23] and targeted multiplex analyte profiling with the Luminex platform [24,25] have yielded molecular tests that are being introduced in clinical laboratories for routine screening purposes. Luminex is a fluorescentbead-based technology that allows the simultaneous 
measurement of multiple analytes in small volume samples. Also, this platform is suitable for further development of accurate, sensitive, and specific assays, given its ease of use in laboratory and clinical settings. The emerging field of mass cytometry [26], which combines the benefits of MS with those of flow cytometry, may also bring new insights into biomarkers of schizophrenia at the functional level, with potential personalized applications in the clinic.

Proteomic studies on serum or plasma from schizophrenia patients have mainly revealed changes in proteins involved in molecular transport (transthyretin [27], apolipoprotein A1 [28]), antioxidant functions (glutathione [29], thioredoxin [30], superoxide dismutase [30]) and growth factors (brain-derived neurotrophic factor [9]), along with changes in inflammation and hormonal signaling that will be described in detail below. Proteomic studies of postmortem brain tissues in psychiatry have identified effects on proteins involved in cellular structure and transport [10], metabolism [11] and synaptic function [12,13], along with several proteins that are also found in serum or plasma, as described above [31]. This suggests that similar pathways might be affected in both the brain and the periphery. However, few of these findings in the brain have been validated and most would be difficult to develop as clinically useful assays due to the inaccessibility of the tissue. The majority of the published studies have used western blot and immunoassay-based methods for validation, which are both dependent on availability of high-affinity and high-specificity antibodies. Other validation methods, such as selective reaction monitoring, have only been used recently in psychiatry [32]. As mentioned in the previous section, in order for a biomarker to achieve clinical status, it is essential that candidates can be cross-validated in large patient cohorts at multiple clinical centers using wellestablished measurement systems [20,21].

\section{Biomarker candidates related to inflammation}

Multiplex immunoassay profiling using cytokine arrays have identified increased levels of IL-1 $\beta$ in cerebrospinal fluid in first-episode schizophrenia patients, suggestive of immune system activation in brain tissue in some patients [33]. This is consistent with studies showing that brain development can be affected by alterations in the balance between pro-inflammatory and anti-inflammatory cytokines $[34,35]$. Furthermore, these immune alterations have been linked to glutamatergic hypofunction, which in turn has been linked to schizophrenia pathophysiology. Transcriptomic and proteomic profiling of schizophrenia postmortem brains have identified increased levels of inflammation-related genes and proteins in oligodendrocytes and endothelial cells, which are known to be regulated by the proinflammatory cytokines
TNF- $\alpha$, IFN- $\alpha$ and IFN- $\gamma[36,37]$. However, such findings could be the result of antipsychotic drug treatment, poor diet or unhealthy life styles, which are often associated with chronic stages of the disease [38].

In addition to the findings in brain tissues, numerous studies have reported peripheral circulatory and cellular pro-inflammatory abnormalities in schizophrenia patients [39]. A meta-analysis of cytokine alterations in schizophrenia showed that molecules such as IL-1 $\beta$, IL-6 and TGF- $\beta$ may be state markers for acute exacerbations, whereas others, including IL-12, IFN- $\gamma$, TNF- $\alpha$ and soluble IL-2 receptor, may be trait markers [40]. By definition, state biomarkers can reflect the changing states or stages of disease and are therefore diagnostic biomarkers. Trait biomarkers are associated with underlying endophenotypes, which can be used to predict the likelihood of developing disease. We carried out multiplex immunoassay analyses of serum from first-episode, anti-psychoticnaive schizophrenia patients that resulted in identification of a disease signature comprising several inflammation-related proteins $[24,25]$ and many of these have been implicated previously in patients with autoimmune diseases [41,42]. Notably, there are studies suggesting that some of the clinical features of schizophrenia may be correlated with autoimmune processes [43]. Autoimmune mechanisms may play a role in the etiology of schizophrenia, as shown by the observation of elevated levels of autoantibodies in blood, cerebrospinal fluid and postmortem brains in some schizophrenia patients [44].

Inflammation in the periphery can affect brain function via effects on the HPA axis. Previous studies have shown that an increase in pro-inflammatory cytokines can lead to altered HPA axis response $[45,46]$. Under normal conditions, corticotrophin releasing factor is released from the hypothalamus, causing adrenocorticotrophic hormone to be released from the pituitary and cortisol to be released from the adrenal cortex [47]. The increase in cortisol has a negative feedback effect on the HPA axis through the brain and pituitary [48] (Figure 1). This mechanism exerts its effects on neurotransmitter systems throughout the brain that can affect mood and behavior.

Recent studies have explored the possibility of using immunomodulatory drugs such as cyclooxygenase-2 inhibitors for treatment of schizophrenia symptoms $[49,50]$. A greater improvement in negative symptoms was observed in early stage patients treated with amisulpride plus celecoxib compared with those treated with amisulpride plus placebo [51]. It should be noted that these findings have not been universally accepted and require validation by testing in separate cohorts. However, it is envisaged that initial screening for patients with high inflammatory status could lead to improved outcomes in similar studies. Aspirin given as adjuvant therapy to regular antipsychotic treatment was used to 


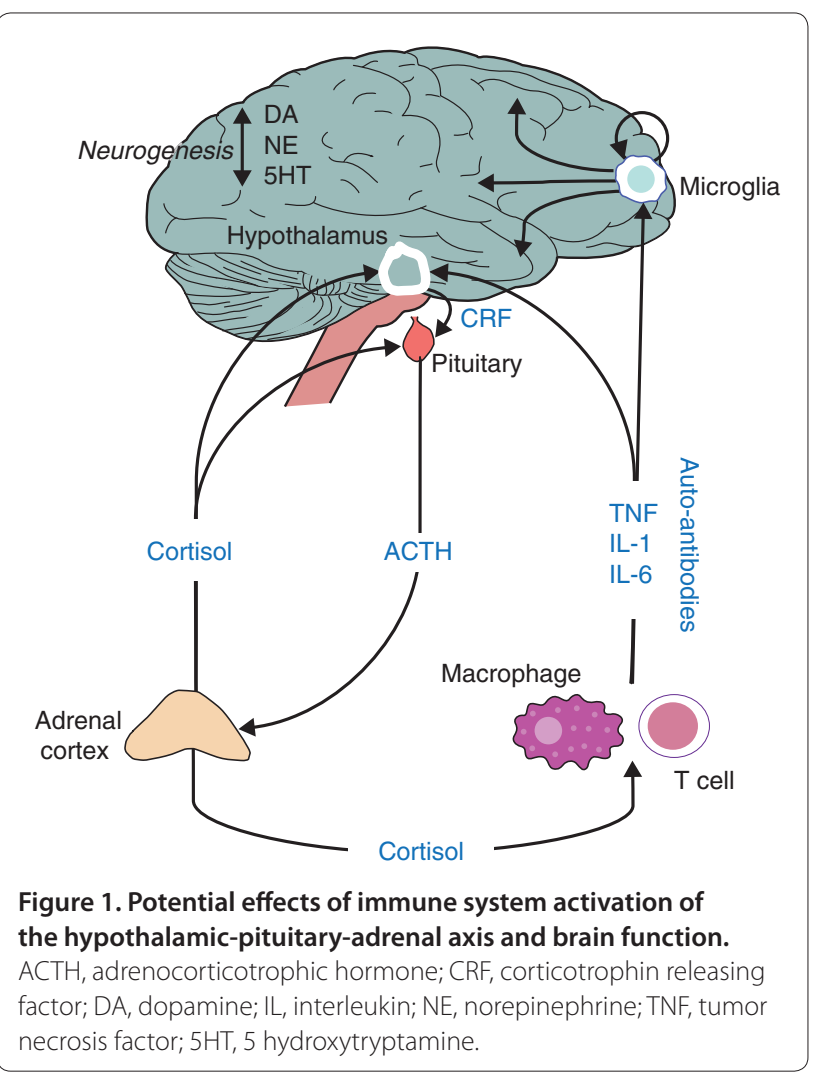

reduce the symptoms associated with schizophrenia spectrum disorders [52].

\section{Proteomic biomarkers related to neuroendocrine pathways}

Recent studies have demonstrated impaired fasting glucose tolerance, high insulin levels and insulin resistance in first episode, antipsychotic naive patients compared with healthy controls $[8,53]$. Hyperinsulinemia and insulin resistance have also been found in drug-free chronic schizophrenia patients [54,55]. Another study found significant hepatic insulin resistance in schizophrenia patients compared with controls using a hyperinsulinemic clamp method [56]. Proteomic profiling of stimulated peripheral blood cells showed altered levels of glycolytic enzymes, the glucose transporter 1 and insulin receptor in cells from first episode antipsychotic-naive schizophrenia patients compared with controls, suggesting an abnormality in glycolysis and other metabolic pathways after immune challenge [57]. In addition, we identified increased levels of circulating insulin-related molecules in first onset schizophrenia subjects, indicating hypersecretion from pancreatic beta cells [16]. This could have important implications since chronically elevated insulin levels can have deleterious effects on brain function [58]. High insulin levels have been associated with aberrant phosphorylation of filamentous proteins, increased brain inflammation and $\beta$-amyloid plaque deposition $[59,60]$. Hyperinsulinemia is also known to perturb the function of neurotransmitter systems [61] and has been shown to have direct effects on hippocampal synaptic plasticity [62]. Moreover, recent imaging studies have shown that patients with impaired glucose tolerance need more insulin than healthy subjects for maximal brain glucose uptake [63]. Therefore, using proteomic biomarkers to substratify patients with metabolic syndrome at early onset of schizophrenia may facilitate early intervention using add-on treatment with antidiabetic agents.

Insulin is co-secreted from pancreatic beta cells with other bioactive peptides, prohormone converting enzymes and accessory proteins in response to elevations in blood glucose [64]. Given that most of these cellular activities are regulated via insulin signaling, it is likely that increased pancreatic beta cell output would affect secretion of hormones and bioactive peptides from other cells throughout the diffuse neuroendocrine system (Figure 2). Consistent with this, increased cortisol secretion and activation of the HPA axis has been identified as a risk factor for schizophrenia in adolescents [65]. Another study showed gender-specific changes in the hormones prolactin, estradiol and testosterone, in first-onset schizophrenia patients [66]. There are also reports of increased levels of arginine vasopressin in schizophrenia patients, which can have profound effects on water excretion [67]. Other studies have also linked abnormal arginine vasopressin levels to changes in mood and behavior [68], which may be mediated through effects on adrenocorticotrophic hormone and cortisol [69].

We carried out multiplex immunoassay analyses of sera from first- and recent-onset schizophrenia patients and found increased levels of insulin, chromogranin A, pancreatic polypeptide, prolactin, progesterone and cortisol, and decreased levels of growth hormone, compared with controls [17]. More recent studies found decreased serum levels of thyroxine, tri-iodothyronine and thyroid stimulating hormone in schizophrenia patients [70]. Since many hormones are influenced by ultradian or circadian rhythms, it is likely that the molecules measured here are co-regulated as part of an oscillatory feedforward-feedback relationship between the pancreatic beta cells, the pituitary and other neuroendocrine components of the HPA and gonadal systems. For instance, high insulin levels have been linked to increased prolactin secretion [71] and impaired pulsatile release of growth hormone [72]. Another study found increased cortisol levels in an antipsychotic-free cohort of schizophrenia patients [73]. Interestingly, initial cortisol levels correlated with negative symptom severity and reduction of negative symptoms was related to altered cortisol levels [74]. The changes in chromogranin 


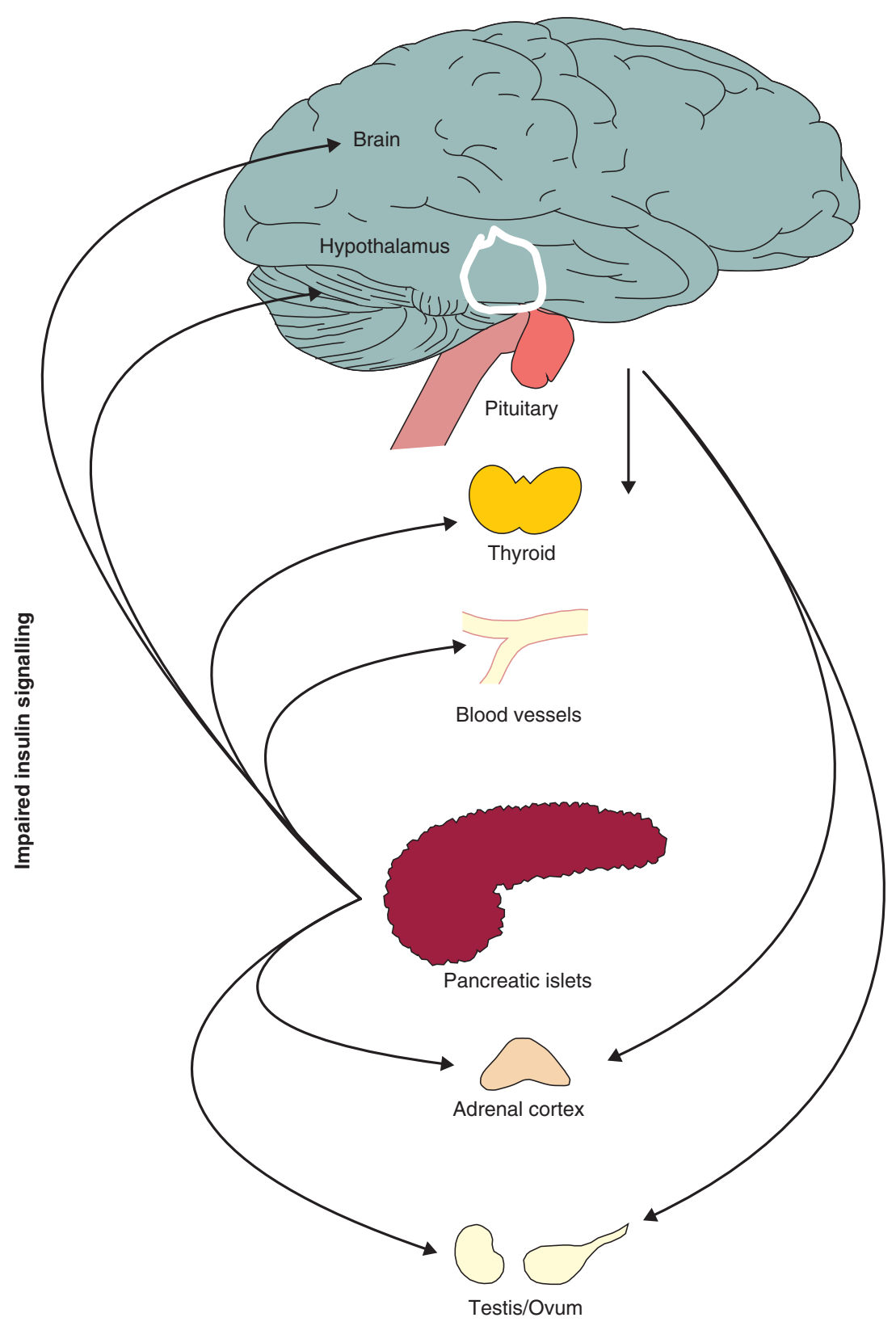

Growth hormone

Arginine vasopressin

Prolactin

Chromogranin A

Pro-opiomelanocortin

Thyroid stimulating hormone

Tri-iodothyronine (T3)

Thyroxine (T4)

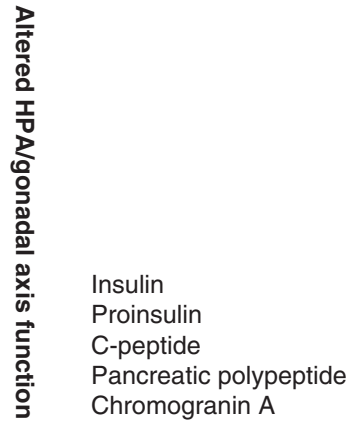

Figure 2. Potential effects of insulin resistance on secretion of other hormones and bioactive molecules over the diffuse neuroendocrine system. HPA, hypothalamic-pituitary-adrenal.

A levels are of interest as this precursor protein undergoes proteolytic processing to produce smaller functional peptides, including vasostatins I and II [75]. This may be important as these peptides regulate the vasodilatation response, which is known to be altered in some schizophrenia patients [76]. Chromogranin A is also processed to form the peptides catestatin and pancreastatin, which inhibit secretion from catecholaminergic adrenal chromaffin cells [77] and pancreatic beta cells [78], respectively. This may also be relevant for schizophrenia, considering the hypothesized role of perturbed catecholamine [79] and insulin signaling pathways [54,55]. Further work is warranted to determine whether other proteins that are secreted via the diffuse neuroendocrine system also have a role in schizophrenia. This could lead to additional insights into the link between central nervous system perturbations and whole body metabolic homeostatic mechanisms.

The finding that high levels of circulating insulinrelated peptides occur in schizophrenia suggests that 
drugs that improve insulin receptor signaling may offer a novel treatment approach. However, antipsychotic drugs are notorious for inducing metabolic side effects such as insulin resistance and weight gain, and the degree of weight gain appears to be linked to therapeutic efficacy. In one study, alterations in body weight, blood glucose and leptin levels were associated with improvement in positive and negative symptoms [80]. Also, changes in levels of serum lipids have been correlated with clinical response to atypical antipsychotic treatment [81]. Therapeutic strategies that target the underlying metabolic dysfunction may provide an effective alternative to treating the traditional neurotransmitter-related endpoint of the disorder. The insulin-sensitizing agents metformin and rosiglitazone have been used to correct the antipsychotic-induced insulin resistance typically associated with this class of drugs, without compromising the psychotropic benefits [82]. We suggest that in future studies testing the effects of such add-on treatments, biomarkers associated with perturbed insulin signaling could be used for patient stratification and for monitoring treatment responses or side effects.

Similar strategies are also being tested for treatment of memory deficits in patients with Alzheimer's disease. Clinical trials are focusing on the use of insulin-sensitizing agents such as rosiglitazone and pioglitazone as an alternative approach to enhance cognition [83]. One group conducted a 6-month, randomized, open-controlled trial in patients with mild Alzheimer's disease accompanied by type 2 diabetes [84]. Patients who received pioglitazone showed improved cognition and increased regional cerebral blood flow, compared with those who received placebo. In addition, other hormones have been targeted as a novel means of treating schizophrenia. For example, the adrenal steroid dehydroepiandrosterone (DHEA) has been used as an add-on therapy in medicated schizophrenia patients, which resulted in a significant improvement in negative, depressive and anxiety symptoms [85]. Notably, improvements in some aspects of psychological function have also been demonstrated following DHEA treatment of patients with Addison's disease [86]. Interestingly, this treatment also led to improvements in self-esteem and mood, indicating their potential utility in psychiatric indications. Also, testing of the selective estrogen receptor modulator raloxifene has been shown to reduce symptoms in postmenopausal schizophrenia women with prominent negative manifestations [87].

\section{Identification of proteomic biomarkers for prediction of treatment response}

Biomarkers that can predict response of schizophrenia subjects prior to treatment would be of major benefit to the patients themselves, as well as to prescribing physicians, clinical scientists, pharmaceutical companies conducting clinical trials and the healthcare services in general. Previous schizophrenia studies have shown that a combination of variants in the histamine 2 receptor gene can predict response to clozapine treatment in $76 \%$ of cases [88]. There is also evidence that genetic variants in dopamine receptors, serotonin receptors and proteins such as catechol-O-methyltransferase, which are involved in drug metabolism pathways and neurotransmitter turnover, can affect clinical response and development of side effects [89]. Another study showed that physical parameters such as waist circumference, body mass index, triglycerides and high-density lipoproteins could be used to predict the antipsychotic-induced development of metabolic syndrome or other insulin resistant states with good sensitivity and specificity [90].

Proteomic studies have shown that schizophrenia patients with higher levels of serum prolactin have a better outcome after 5 years of antipsychotic treatment [91]. We carried out multiplex immunoassay profiling of serum and found that the levels of seven proteins (IL-16, fatty acid binding protein, ferritin, C-reactive protein, myoglobin, prolactin and complement factor $\mathrm{H}$ ) could predict improvement in positive symptoms, and two proteins (matrix metalloproteinase 2 and insulin) could be used for prediction of improved negative symptoms in first-onset schizophrenia patients after antipsychotic treatment [92]. In addition, we found that reduced levels of insulin and leptin and increased levels of TGF- $\beta$ were indicative of impending relapse in the same antipsychotic-treated patients.

Other studies have shown that a number of serum molecules are altered in response to antipsychotic treatment that have associations with inflammatory [40] and hormonal [92,93] pathways. Thus, many of these could be used as potential biomarkers of efficacy or side-effect response. Further studies investigating these molecules may lead to molecular tests that can aid in the identification of patients who are likely to respond to specific antipsychotic treatments and who may benefit from adjunct therapies targeting either inflammatory or metabolic pathways. This could also offer the chance for clinicians to take appropriate action such as patient assessment, counseling and re-adjusting medications according to experimentally determined biomarker profiles. In some cases this could mean switching patients to a different antipsychotic with different efficacy or sideeffect profile.

\section{Conclusions}

The studies presented here indicate considerable progress in using proteomic-based biomarkers for the ultimate goal of improving care of schizophrenia patients. We also suggest that there is critical need for integrating data 


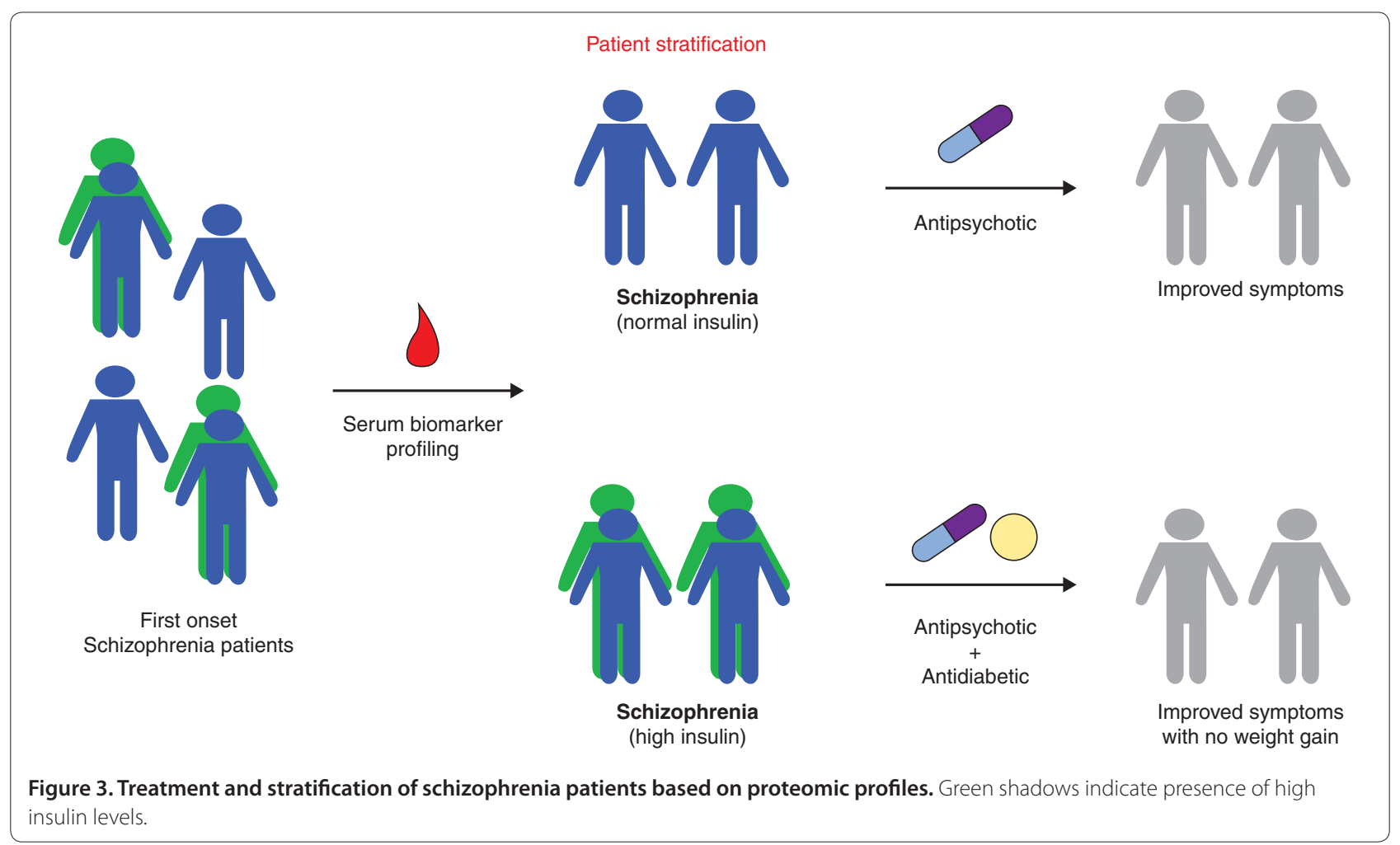

from a number of different molecular profiling methods such as proteomic, transcriptomic and metabonomic platforms. This could lead to a more integrative view of the perturbed biological pathways in schizophrenia through a systems biology approach. Biomarker fingerprints have been identified in serum samples of firstonset schizophrenia patients; this indicates that this condition may comprise different subtypes at the molecular level, which could potentially lead to earlier and more targeted intervention, reducing disease progression. Many patients have patterns of circulating molecules suggestive of dysfunctional immune or metabolic systems, with elevated levels of inflammatory cytokines or metabolic abnormalities such as high insulin levels or insulin resistance. However, it is possible that even these are not distinct subtypes but may be related to the same underlying phenomena. Nevertheless, better classification of patients based on such molecular profiles would enable stratification prior to treatment. This would entail adopting an endophenotype approach by using specific readouts such as inflammation or insulin resistance as end-point measurements. Furthermore, a molecular test that recognizes such subtypes may be used for identifying patients who are most likely to respond to particular treatments. This could lead to more effective treatments with fewer side effects. For example, patients with high insulin levels could be administered insulin sensitizing agents as an adjunct therapy with standard antipsychotics, aiming to minimize metabolic side effects while maintaining improved psychotherapeutic responses (Figure 3). This approach has already shown promise in clinical studies that have used metformin or rosiglitazone to minimize the weight gain in response to treatment with typical and atypical antipsychotics $[82,94]$.

Thus far, none of the longitudinal studies that has targeted the inflammatory or metabolic components in schizophrenia has incorporated the use of biomarkers to stratify patient populations either prior to or during clinical trials. Therefore, it will be necessary to explore the relationship between alterations that appear to be intrinsic to schizophrenia etiology and to assess the response to currently used antipsychotics. Furthermore, the increased use of longitudinal studies will help to address one of the key requirements of proteomic-based biomarkers, which is the need for stability over time. This could also be assessed in controls as a means of identifying factors that can affect biomarker readouts.

The categorization of patients based on proteomic biomarker profiles for optimized treatment is a form of personalized medicine. This important paradigm has probably been best exemplified in the field of breast cancer, where the presence of the human epidermal growth factor receptor 2 (HER-2) helps to select those patients who will benefit from treatment with Herceptin [95]. Such efforts will lead to novel therapeutic targets for drug development and to the individualization of 
treatment, maximizing the likelihood of positive therapeutic outcomes. In the case of schizophrenia it also offers the opportunity to support the personalized medicine vision by de-convoluting the complexity of the disease from superficial symptom characterization to an objective biological understanding. In particular, proteomic methods could be used to determine whether patients can be divided into subgroups that show distinct changes in either immune or metabolic pathways, as opposed to mixed changes in both pathways. This would help to address the hypothesis that schizophrenia is a heterogeneous condition comprising distinct subtypes and could also lead to the development of much-needed novel therapeutic approaches for schizophrenia that target the affected pathways.

\section{Abbreviations}

DHEA, dehydroepiandrosterone; FDA, Food and Drug Administration; HPA, hypothalamic-pituitary-adrenal; IFN, interferon; IL, interleukin; MS, mass spectrometry; TGF, transforming growth factor; TNF, tumor necrosis factor.

\section{Competing interests}

PCG, ES and SB are consultants for Myriad-RBM. All other authors declare that they have no competing interests.

\section{Acknowledgements}

This work was supported by funding from the Stanley Medical Research Institute, the European Union FP7 SchizDX research programme (grant reference 223427) and the Dutch Fund for Economic Structure Reinforcement (FES), under grant agreement number 0908 (NeuroBasic PharmaPhenomics project).

\section{Author details}

'Department of Chemical Engineering and Biotechnology, University of Cambridge, Tennis Court Road, Cambridge, CB2 1QT, UK. ${ }^{2}$ Max Planck Institute of Psychiatry, Proteomics and Biomarkers, Kraepelinstr. 2-10 80804, Munich, Germany. ${ }^{3}$ Department of Psychiatry, Ludwig-MaximiliansUniversity (LMU), Nussbaumstr. 7, 80336, Munich, Germany. ${ }^{4}$ Laboratório de Neurociências (LIM-27), Instituto de Psiquiatria, Faculdade de Medicina, Universidade de São Paulo, CP $809105403-010$ São Paulo - SP - Brasil. ${ }^{5}$ Department of Neuroscience, Erasmus Medical Centre, NL-3000 CA Rotterdam, The Netherlands

\section{Published: X Month 2013}

\section{References}

1. Chakos M, Lieberman J, Hoffman E, Bradford D, Sheitman B: Effectiveness of second-generation antipsychotics in patients with treatment-resistant schizophrenia: a review and meta-analysis of randomized trials. Am $J$ Psychiatry 2001, 158:518-526.

2. Wong EH, Yocca F, Smith MA, Lee CM: Challenges and opportunities for drug discovery in psychiatric disorders: the drug hunters' perspective. Int J Neuropsychopharmacol 2010, 13:1269-1284.

3. Lovatt A, Mason O, Brett C, Peters E: Psychotic-like experiences, appraisals, and trauma. J Nerv Ment Dis 2010, 198:813-819.

4. Izmailov R, Guest PC, Bahn S, Schwarz E: Algorithm development for diagnostic biomarker assays. Int Rev Neurobio/ 2011, 101:279-298.

5. Muller N, Schwarz M: Schizophrenia as an inflammation-mediated dysbalance of glutamatergic neurotransmission. Neurotox Res 2006, 10:131-148.

6. Martins-de-Souza D, Gattaz WF, Schmitt A, Rewerts C, Maccarrone G, DiasNeto E, Turck CW: Prefrontal cortex shotgun proteome analysis reveals altered calcium homeostasis and immune system imbalance in schizophrenia. Eur Arch Psychiatry Clin Neurosci 2009, 259:151-163.

7. Hasnain M, Fredrickson SK, Vieweg WV, Pandurangi AK: Metabolic syndrome associated with schizophrenia and atypical antipsychotics. Curr Diab Rep 2010, 10:209-216
8. Ryan MC, Collins $\mathrm{P}$, Thakore $\mathrm{JH}$ : Impaired fasting glucose tolerance in firstepisode, drug-naive patients with schizophrenia. Am J Psychiatry 2003, 160:284-289.

9. Wu Z, Zhang XY, Wang H, Tang W, Xia Y, Zhang F, Liu J, Fu Y, Hu J, Chen Y, Liu L, Chen da C, Xiu MH, Kosten TR, He J: Elevated plasma superoxide dismutase in first-episode and drug naive patients with schizophrenia: inverse association with positive symptoms. Prog Neuropsychopharmacol Biol Psychiatry 2012, 36:34-38.

10. Carlino D, Leone E, Di Cola F, Baj G, Marin R, Dinelli G, Tongiorgi E, De Vanna M: Low serum truncated-BDNF isoform correlates with higher cognitive impairment in schizophrenia. J Psychiatr Res 2011, 45:273-279.

11. English JA, Dicker P, Föcking M, Dunn MJ, Cotter DR: 2-D DIGE analysis implicates cytoskeletal abnormalities in psychiatric disease. Proteomics 2009, 9:3368-3382.

12. Föcking M, Dicker P, English JA, Schubert KO, Dunn MJ, Cotter DR: Common proteomic changes in the hippocampus in schizophrenia and bipolar disorder and particular evidence for involvement of cornu ammonis regions 2 and 3. Arch Gen Psychiatry 2011, 68:477-488.

13. Pennington $K$, Beasley CL, Dicker P, Fagan A, English J, Pariante CM, Wait R, Dunn MJ, Cotter DR: Prominent synaptic and metabolic abnormalities revealed by proteomic analysis of the dorsolateral prefrontal cortex in schizophrenia and bipolar disorder. Mol Psychiatry 2008, 13:1102-1117.

14. Chan MK, Tsang TM, Harris LW, Guest PC, Holmes E, Bahn S: Evidence for disease and antipsychotic medication effects in post-mortem brain from schizophrenia patients. Mol Psychiatry 2011, 16:1189-1202.

15. Martins-de-Souza D, Harris LW, Guest PC, Bahn S: The role of energy metabolism dysfunction and oxidative stress in schizophrenia revealed by proteomics. Antioxid Redox Signal 2011, 15:2067-2079.

16. Guest PC, Wang L, Harris LW, Burling K, Levin Y, Ernst A, Wayland MT, Umrania Y, Herberth M, Koethe D, van Beveren JM, Rothermundt M, McAllister G, Leweke FM, Steiner J, Bahn S: Increased levels of circulating insulin-related peptides in first-onset, antipsychotic naïve schizophrenia patients. $\mathrm{Mol}$ Psychiatry 2010, 15:118-119.

17. Guest PC, Schwarz E, Krishnamurthy D, Harris LW, Leweke FM, Rothermundt M, van Beveren NJ, Spain M, Barnes A, Steiner J, Rahmoune H, Bahn S: Altered levels of circulating insulin and other neuroendocrine hormones associated with the onset of schizophrenia. Psychoneuroendocrinology 2011, 36:1092-1096.

18. Laughren TP: What's next after 50 years of psychiatric drug development: an FDA perspective. J Clin Psychiatry 2010, 71:1196-1204.

19. Ovens J: Funding for accelerating drug development initiative critical. Nat Rev Drug Discov 2006, 5:271

20. Goodsaid F, Frueh FW: Implementing the U.S. FDA guidance on pharmacogenomic data submissions. Environ Mol Mutagen 2007, 48:354-358.

21. Goodsaid F, Frueh F: Process map proposal for the validation of genomic biomarkers. Pharmacogenomics 2006, 7:773-782.

22. Filiou MD, Martins-de-Souza D, Guest PC, Bahn S, Turck CW: To label or not to label: applications of quantitative proteomics in neuroscience research Proteomics 2012, 12:736-747.

23. Levin Y, Wang L, Schwarz E, Koethe D, Leweke FM, Bahn S: Global proteomic profiling reveals altered proteomic signature in schizophrenia serum. $\mathrm{Mo}$ Psychiatry 2010, 15:1088-1100.

24. Schwarz E, Izmailov R, Spain M, Barnes A, Mapes JP, Guest PC, Rahmoune H, Pietsch S, Leweke FM, Rothermundt M, Steiner J, Koethe D, Kranaster L, Ohrmann P, Suslow T, Levin Y, Bogerts B, van Beveren NJ, McAllister G, Weber N, Niebuhr D, Cowan D, Yolken RH, Bahn S: Validation of a blood-based laboratory test to aid in the confirmation of a diagnosis of schizophrenia. Biomark Insights 2010, 5:39-47.

25. Schwarz E, Guest PC, Rahmoune H, Harris LW, Wang L, Leweke FM, Rothermundt M, Bogerts B, Koethe D, Kranaster L, Ohrmann P, Suslow T, McAllister G, Spain M, Barnes A, van Beveren NJ, Baron-Cohen S, Steiner J, Torrey FE, Yolken RH, Bahn S: Identification of a biological signature for schizophrenia in serum. Mol Psychiatry 2012, 17:494-502.

26. Bendall SC, Simonds EF, Qiu P, Amir el-AD, Krutzik PO, Finck R: Single-cell mass cytometry of differential immune and drug responses across a human hematopoietic continuum. Science 2011, 332:687-696.

27. Wan C, Yang Y, Li H, La Y, Zhu H, Jiang L, Chen Y, Feng G, He L: Dysregulation of retinoid transporters expression in body fluids of schizophrenia patients. J Proteome Res 2006, 5:3213-3216.

28. La YJ, Wan CL, Zhu H, Yang YF, Chen YS, Pan YX, Feng GY, He L: Decreased 
levels of apolipoprotein A-I in plasma of schizophrenic patients. J Neural Transm 2007, 114:657-663.

29. Raffa M, Atig F, Mhalla A, Kerkeni A, Mechri A: Decreased glutathione levels and impaired antioxidant enzyme activities in drug-naive first-episode schizophrenic patients. BMC Psychiatry 2011, 11:124.

30. Zhang XY, Chen da C, Xiu MH, Wang F, Qi LY, Sun HQ, Chen S, He SC, Wu GY, Haile CN, Kosten TA, Lu L, Kosten TR: The novel oxidative stress marker thioredoxin is increased in first-episode schizophrenic patients. Schizophr Res 2009, 113:151-157.

31. Harris LW, Pietsch S, Cheng TM, Schwarz E, Guest PC, Bahn S: Comparison of peripheral and central schizophrenia biomarker profiles. PLoS One 2012, 7:e46368.

32. Martins-de-Souza D, Guest PC, Harris LW, Vanattou-Saifoudine N, Webster MJ, Rahmoune H, Bahn S: Identification of proteomic signatures associated with depression and psychotic depression in post-mortem brains from major depression patients. Trans/ Psychiatry 2012, 2:e87.

33. Söderlund J, Schröder J, Nordin C, Samuelsson M, Walther-Jallow L, Karlsson $H$, Erhardt S, Engberg G: Activation of brain interleukin-1 beta in schizophrenia. Mol Psychiatry 2009, 14:1069-1071.

34. Merrill JE: Tumor necrosis factor alpha, interleukin 1 and related cytokines in brain development: normal and pathological. Dev Neurosci 1992, 14:1-10.

35. Meyer U, Feldon J, Yee BK: A review of the fetal brain cytokine imbalance hypothesis of schizophrenia. Schizophr Bull 2009, 35:959-972.

36. Saetre P, Emilsson L, Axelsson E, Kreuger J, Lindholm E, Jazin E: Inflammationrelated genes up-regulated in schizophrenia brains. BMC Psychiatry 2007, 7:46.

37. Schmitt A, Leonardi-Essmann F, Durrenberger PF, Parlapani E, SchneiderAxmann T, Spanagel R, Arzberger T, Kretzschmar H, Herrera-Marschitz M, Gruber O, Reynolds R, Falkai P, Gebicke-Haerter PJ: Regulation of immunemodulatory genes in left superior temporal cortex of schizophrenia patients: a genome-wide microarray study. World J Biol Psychiatry 2011, 12:201-215

38. Montejo AL: The need for routine physical health care in schizophrenia. Eur Psychiatry 2010, 25(Suppl 2):S3-5.

39. Pedrini M, Massuda R, Fries GR, de Bittencourt Pasquali MA, Schnorr CE, F Moreira JC, Teixeira AL, Lobato MI, Walz JC, Belmonte-de-Abreu PS, KauerSant'Anna M, Kapczinski F, Gama CS: Similarities in serum oxidative stress markers and inflammatory cytokines in patients with overt schizophrenia at early and late stages of chronicity. J Psychiatr Res 2012, 46:819-824.

40. Miller BJ, Buckley P, Seabolt W, Mellor A, Kirkpatrick B: Meta-analysis of cytokine alterations in schizophrenia: clinical status and antipsychotic effects. Biol Psychiatry 2011, 70:663-671.

41. Fessel WJ, Solomon GF: Psychosis and systemic lupus erythematosus: a review of the literature and case reports. Calif Med 1960, 92:266-270.

42. Eaton WW, Byrne M, Ewald H, Mors O, Chen CY, Agerbo E, Mortensen PB: Association of schizophrenia and autoimmune diseases: linkage of Danish national registers. Am J Psychiatry 2006, 163:521-528.

43. Jones AL, Mowry BJ, Pender MP, Greer JM: Immune dysregulation and selfreactivity in schizophrenia: do some cases of schizophrenia have an autoimmune basis? Immunol Cell Biol 2005, 83:9-17.

44. Sirota P, Firer MA, Schild K, Tanay A, Elizur A, Meytes D, Slor H: Autoantibodies to DNA in multicase families with schizophrenia. Biol Psychiatry 1993, 33:450-455.

45. Späth-Schwalbe E, Born J, Schrezenmeier H, Bornstein SR, Stromeyer P, Drechsler S, Fehm HL, Porzsolt F: Interleukin-6 stimulates the hypothalamus-pituitary-adrenocortical axis in man. $J$ Clin Endocrinol Metab 1994, 79:1212-1214

46. Straub RH, Buttgereit F, Cutolo M: Alterations of the hypothalamic-pituitaryadrenal axis in systemic immune diseases - a role for misguided energy regulation. Clin Exp Rheumatol 2011, 29:S23-31.

47. Bremner JD: Traumatic stress: effects on the brain. Dialogues Clin Neurosci 2006, 8:445-461

48. Spijker AT, van Rossum EF: Glucocorticoid sensitivity in mood disorders. Neuroendocrinology 2012, 95:179-186.

49. Müller N, Riedel M, Schwarz MJ: Psychotropic effects of COX-2 inhibitors a possible new approach for the treatment of psychiatric disorders. Pharmacopsychiatry 2004, 37:266-269.

50. Akhondzadeh S, Tabatabaee M, Amini H, Ahmadi Abhari SA, Abbasi SH, Behnam B: Celecoxib as adjunctive therapy in schizophrenia: a doubleblind, randomized and placebo-controlled trial. Schizophr Res 2007
90:179-185.

51. Müller N, Krause D, Dehning S, Musil R, Schennach-Wolff R, Obermeier M, Möller HJ, Klauss V, Schwarz MJ, Riedel M: Celecoxib treatment in an early stage of schizophrenia: results of a randomized, double-blind, placebocontrolled trial of celecoxib augmentation of amisulpride treatment. Schizophr Res 2010, 121:118-124.

52. Laan W, Grobbee DE, Selten JP, Heijnen CJ, Kahn RS, Burger H: Adjuvant aspirin therapy reduces symptoms of schizophrenia spectrum disorders: results from a randomized, double-blind, placebo-controlled trial. J Clin Psychiatry 2010, 71:520-527.

53. Spelman LM, Walsh PI, Sharifi N, Collins P, Thakore JH: Impaired glucose tolerance in first-episode drug-naive patients with schizophrenia. Diabet Med 2007, 24:481-485.

54. Arranz B, Rosel P, Ramirez N, Duenas R, Fernandez P, Sanchez JM, Navarro MA San L: Insulin resistance and increased leptin concentrations in noncompliant schizophrenia patients but not in antipsychotic-naive firstepisode schizophrenia patients. J Clin Psychiatry 2004, 65:1335-1342.

55. Cohn TA, Remington G, Zipursky RB, Azad A, Connolly P, Wolever TM: Insulin resistance and adiponectin levels in drug-free patients with schizophrenia: A preliminary report. Can J Psychiatry 2006, 51:382-386.

56. van Nimwegen LJ, Storosum JG, Blumer RM, Allick G, Venema HW, de Haan L, Becker H, van Amelsvoort T, Ackermans MT, Fliers E, Serlie MJ, Sauerwein HP: Hepatic insulin resistance in antipsychotic naive schizophrenic patients: stable isotope studies of glucose metabolism. J Clin Endocrinol Metab 2008, 93:572-577.

57. Herberth M, Koethe D, Cheng TM, Krzyszton ND, Schoeffmann S, Guest PC, Rahmoune H, Harris LW, Kranaster L, Leweke FM, Bahn S: Impaired glycolytic response in peripheral blood mononuclear cells of first-onset antipsychotic-naive schizophrenia patients. Mol Psychiatry 2011 16:848-859.

58. Taguchi A, Wartschow LM, White MF: Brain IRS2 signaling coordinates life span and nutrient homeostasis. Science 2007, 317:369-372.

59. Convit A: Links between cognitive impairment in insulin resistance: an explanatory model. Neurobiol Aging 2005, 26(Suppl 1):31-35.

60. Craft S: Insulin resistance and Alzheimer's disease pathogenesis: potential mechanisms and implications for treatment. Curr Alzheimer Res 2007 4:147-152.

61. Bello NT, Hajnal A: Alterations in blood glucose levels under hyperinsulinemia affect accumbens dopamine. Physiol Behav 2006, 88:138-145.

62. O'Malley D, Shanley $L$, Harvey J: Insulin inhibits rat hippocampal neurones via activation of ATP-sensitive $\mathrm{K}^{+}$and large conductance $\mathrm{Ca}^{2+}$-activated $\mathrm{K}^{+}$ channels. Neuropharmacology 2003, 44:855-863.

63. Hirvonen J, Virtanen KA, Nummenmaa L, Hannukainen JC, Honka M-J, Bucci M, Nesterov SV, Parkkola R, Rinne J, lozzo P, Nuutila P: Effects of insulin on brain glucose metabolism in impaired glucose tolerance. Diabetes 2011, 60:443-447.

64. Hutton JC: Insulin secretory granule biogenesis and the proinsulinprocessing endopeptidases. Diabetologia 1994, 37(Suppl 2):S48-56.

65. Corcoran CM, Smith C, McLaughlin D, Auther A, Malaspina D, Cornblatt B: HPA axis function and symptoms in adolescents at clinical high risk for schizophrenia. Schizophr Res 2012, 135:170-174.

66. Gorobets LN, Matrosova MI: Specialties of prolactin secretion and peripheral reproductive sex hormones in patients with of first episode of schizophrenia. Zh Nevrol Psikhiatr Im S S Korsakova 2010, 110:17-22.

67. Goldman MB: The mechanism of life-threatening water imbalance in schizophrenia and its relationship to the underlying psychiatric illness. Brain Res Rev 2009, 61:210-220.

68. Heinrichs M, von Dawans B, Domes G: Oxytocin, vasopressin, and human social behavior. Front Neuroendocrinol 2009, 30:548-557.

69. Ryan MC, Sharifi N, Condren R, Thakore JH: Evidence of basal pituitaryadrenal overactivity in first episode, drug naive patients with schizophrenia. Psychoneuroendocrinology 2004, 29:1065-1070.

70. Akiibinu MO, Ogundahunsi OA, Ogunyemi EO: Inter-relationship of plasma markers of oxidative stress and thyroid hormones in schizophrenics. BMC Res Notes 2012, 5:169.

71. Ben-Jonathan N, Hugo ER, Brandebourg TD, LaPensee CR: Focus on prolactin as a metabolic hormone. Trends Endocrinol Metab 2006, 17:110-116.

72. Tannenbaum GS, Martin JB, Colle E: Ultradian growth hormone rhythm in the rat: effects of feeding, hyperglycemia, and insulin-induced hypoglycemia. Endocrinology 1976, 99:720-727. 
73. Meltzer HY, Lee MA, Jayathilake $K$ : The blunted plasma cortisol response to apomorphine and its relationship to treatment response in patients with schizophrenia. Neuropsychopharmacology 2001, 24:278-290.

74. Zhang XY, Zhou DF, Cao LY, Wu GY, Shen YC: Cortisol and cytokines in chronic and treatment-resistant patients with schizophrenia: association with psychopathology and response to antipsychotics. Neuropsychopharmacology 2005, 30:1532-1538.

75. Helle KB, Corti A, Metz-Boutigue MH, Tota B: The endocrine role for chromogranin $\mathrm{A}$ : a prohormone for peptides with regulatory properties. Cell Mol Life Sci 2007, 64:2863-2886.

76. Nilsson BM, Hultman CM, Wiesel FA: Niacin skin-flush response and electrodermal activity in patients with schizophrenia and healthy controls. Prostaglandins Leukot Essent Fatty Acids 2006, 74:339-346.

77. Garcia GE, Gabbai FB, O'Connor DT, Dinh TQ, Kennedy B, Ziegler MG, Takiyyuddin MA: Does chromostatin influence catecholamine release or blood pressure in vivo? Peptides 1994, 15:195-197.

78. Tatemoto K, Efendić S, Mutt V, Makk G, Feistner GJ, Barchas JD: Pancreastatin, a novel pancreatic peptide that inhibits insulin secretion. Nature 1986, 324:476-478.

79. Meltzer HY, Stahl SM. The dopamine hypothesis of schizophrenia: a review. Schizophr Bull 1976, 2:19-76.

80. Meltzer HY, Perry E, Jayathilake K: Clozapine-induced weight gain predicts improvement in psychopathology. Schizophr Res 2003, 59:19-27.

81. Huang TL, Chen JF: Serum lipid profiles and schizophrenia: effects of conventional or atypical antipsychotic drugs in Taiwan. Schizophr Res 2005, 80:55-59.

82. Bahtiyar G, Weiss K, Sacerdote AS: Novel endocrine disrupter effects of classic and atypical antipsychotic agents and divalproex: induction of adrenal hyperandrogenism, reversible with metformin or rosiglitazone. Endocr Pract 2007, 13:601-608.

83. Sato $T$, Hanyu H, Hirao K, Kanetaka H, Sakurai H, Iwamoto T: Efficacy of PPARgamma agonist pioglitazone in mild Alzheimer disease. Neurobiol Aging 2011, 32:1626-1633.

84. Landreth G, Jiang Q, Mandrekar S, Heneka M: PPARgamma agonists as therapeutics for the treatment of Alzheimer's disease. Neurotherapeutics 2008, 5: 481-489.

85. Nachshoni T, Ebert T, Abramovitch Y, Assael-Amir M, Kotler M, Maayan R, Weizman A, Strous RD: Improvement of extrapyramidal symptoms following dehydroepiandrosterone (DHEA) administration in antipsychotic treated schizophrenia patients: a randomized, double-blind placebo controlled trial. Schizophr Res 2005, 79:251-256.
86. Hunt PJ, Gurnell EM, Huppert FA, Richards C, Prevost AT, Wass JA, Herbert J, Chatterjee VK: Improvement in mood and fatigue after dehydroepiandrosterone replacement in Addison's disease in a randomized, double blind trial. J Clin Endocrinol Metab 2000, 85:4650-4656.

87. Usall J, Huerta-Ramos E, Iniesta R, Cobo J, Araya S, Roca M, Serrano-Blanco A, Teba F, Ochoa S: Raloxifene as an adjunctive treatment for postmenopausal women with schizophrenia: a double-blind, randomized, placebo-controlled trial. J Clin Psychiatry 2011, 72:1552-1557.

88. Arranz MJ, Munro J, Birkett J, Bolonna A, Mancama D, Sodhi M, Lesch KP, Meyer JF, Sham P, Collier DA, Murray RM, Kerwin RW: Pharmacogenetic prediction of clozapine response. Lancet 2000, 355:1615-1616.

89. Zhang JP, Malhotra AK: Pharmacogenetics and antipsychotics: therapeutic efficacy and side effects prediction. Expert Opin Drug Metab Toxicol 2011, 7:9-37.

90. Jin H, Meyer J, Mudaliar S, Henry R, Khandrika S, Glorioso DK, Kraemer H, Jeste D: Use of clinical markers to identify metabolic syndrome in antipsychotictreated patients. J Clin Psychiatry 2010, 71:1273-1278.

91. Shrivastava A, Johnston M, Bureau Y, Shah N: Baseline serum prolactin in drug-naive, first-episode schizophrenia and outcome at five years: is it a predictive factor? Innov Clin Neurosci 2012, 9:17-21.

92. Schwarz E, Guest PC, Steiner J, Bogerts B, Bahn S: Identification of bloodbased molecular signatures for prediction of response and relapse in schizophrenia patients. Transl Psychiatry 2012, 2:e82.

93. Raposo NR, Ferreira AS, Gattaz WF: Body mass index increase, serum leptin, adiponectin, neuropeptide $Y$ and lipid levels during treatment with olanzapine and haloperidol. Pharmacopsychiatry 2011, 44:169-172.

94. Newall H, Myles N, Ward PB, Samaras K, Shiers D, Curtis J: Efficacy of metformin for prevention of weight gain in psychiatric populations: a review. Int Clin Psychopharmacol 2012, 27:69-75.

95. Demonty G, Bernard-Marty C, Puglisi F, Mancini I, Piccart M: Progress and new standards of care in the management of HER-2 positive breast cancer. Eur J Cancer 2007, 43:497-509.

doi:10.1186/gm429

Cite this article as: Guest PC, et al:: Proteomic profiling in schizophrenia: enabling stratification for more effective treatment. Genome Medicine 2013, $5: 25$ 